\title{
Navigation in the water maze: The role of proximal and distal visual cues, path integration, and magnetic field information
}

\author{
DONALD P. CAIN, JASON BEIKO, and FRANCIS BOON \\ University of Western Ontario, London, Ontario, Canada
}

\begin{abstract}
The kind of information used in acquisition of the water maze task is an important element in the hypotheses generated to explain this form of learning. Therefore, we examined the role of proximal maze wall cue, distal visual cue, path integration, and direction-static magnetic field information in the acquisition of this task by male hooded rats. The availability of proximal cue and directionstatic magnetic field information failed to support place responses when distal visual cues were occluded by black curtains and the rats were prevented from knowing the point of entry into the pool or using path integration information. When distal visual cues were occluded, the rats could make use of path integration information to perform a place response. Place responding was most robust when both distal visual cues and path integration information were available.
\end{abstract}

The learning of a place response in the water maze task (Morris, 1984) is one of the most rapidly acquired types of navigation learning. Typically, rats can learn to swim to a hidden escape platform from any point around the rim of a pool in fewer than 10 trials. Although it is known that large landmark cues on the maze pool wall near the escape platform (Kolb, Buhrmann, McDonald, \& Sutherland, 1994) and distal visual cues outside of the maze pool (Morris, 1981, 1984; Sutherland, Whishaw, \& Regehr, 1982) can play a major role in this form of learning, it is not known definitively whether other sources of information also contribute. For example, a practical question is whether a smaller cue, such as a mark or blemish located on the maze wall in constant relation to the platform, could serve as a basis for navigation.

Another potential source of information is path integration, a mechanism thought to involve the self-monitoring of movement (Etienne, 1987; McNaughton et al., 1996; Whishaw, McKenna, \& Maaswinkel, 1997). Some researchers have proposed that path integration mechanisms may be required for the development of landmark-based navigation (Gallistel, 1990; McNaughton, Chen, \& Markus, 1991). Although mammals can use path integration to navigate in radial arm maze and food-carrying tasks (Dudchenko, Goodridge, Seiterle, \& Taube, 1997; Etienne, 1987; Martin, Harley, Smith, Hoyles, \& Hynes, 1997; Whishaw et al., 1997), evidence from recent studies suggest that path integration is not required in the water maze task or

This research was supported by a grant from NSERC to D.P.C. The authors thank Martin Kavaliers and David Sherry for helpful comments on the manuscript. Correspondence should be addressed to D. P. Cain, Department of Psychology, Graduate Program in Neuroscience, University of Western Ontario, London, ON, Canada N6A 5C2 (e-mail: cain@sscl.uwo.ca). in a water version of the radial arm maze task (Dudchenko et al., 1997; Martin et al., 1997). In one of the studies (Dudchenko et al., 1997), distal cues were eliminated by a white curtain that surrounded the pool during training, and only a proximal cue consisting of a large $\left(63^{\circ}\right.$ of arc) black sheet attached to the inside of the curtain was available. Since it is possible that path integration may be involved in tasks that depend on many distal cues (as might be expected in the wild) but may not be required in tasks that involve one large proximal cue (as in Dudchenko et al., 1997), it is important to evaluate the role of path integration in a conventional water maze task that requires the use of many distal cues. Martin et al. (1997) concluded that path integration "or other cue sources, not obvious to the experimenters, were available for problem solution" (p. 190). Thus, the exact sources of information or navigation mechanisms involved in the water maze task have not been definitively identified.

A final source of information that might be relevant to navigation in the water maze is magnetic field information (Potegal, 1987). There is evidence that, in vertebrates, the earth's magnetic field may serve as a source of both directional (i.e., "compass") and geographic position (i.e., "map") information (Wiltschko \& Wiltschko, 1996). A variety of rodent species have been shown to be sensitive to magnetic fields, and it has been proposed that they might make use of magnetic field information in navigation (August, Ayvazian, \& Anderson, 1989; Burda, Marhold, Westenberger, Wiltschko, \& Wiltschko, 1990; Chafetz, 1982; Mather, 1985; Mather \& Baker, 1981; Olcese, Reuss, \& Semm, 1988). Recent studies with deer mice and meadow voles suggest that low-frequency $(60 \mathrm{~Hz})$ magnetic fields can provide directional cues in the water maze task (Kavaliers, Eckel, \& Ossenkopp, 1993; Kavaliers et al., 1996). However, the possible role of directional static magnetic 
fields, which more closely approximate the geomagnetic field, as orientation or navigation cues has not been examined in the water maze task.

Thus, there are at least four possible sources of information for navigation in the water maze: proximal maze wall cues, distal visual cues, path integration, and directionstatic magnetic field information. No experiment has evaluated the role of these sources of information in the water maze task in the same rats. This was the goal of the present study. This issue is important in the context of suggestions that rats use numerous navigation strategies concurrently in the water maze (Whishaw \& Mittleman, 1986).

This research was conducted in a large university building with electrical lines, air conditioning equipment, and other common sources of magnetic fields on the same or nearby floors. These potential sources of magnetic field information or distortion might complicate the analysis, relative to the situation in the wild. However, with one exception (Kavaliers \& Galea, 1994), all water maze experiments to date appear to have been conducted in modern buildings with comparable sources of background magnetic field information and distortion of the geomagnetic field. Therefore, we attempted to approach the question of directional magnetic cues in an experimental setting that is representative of typical water maze experiments as reported in the research literature.

\section{GENERAL METHOD}

\section{Subjects}

The subjects were male Long-Evans hooded rats weighing $275-350 \mathrm{~g}$. They were housed individually, fed ad lib, and tested during the light phase of a 12 -h light:dark cycle. All rats were naive when testing began and were gentled prior to the start of training.

\section{Apparatus}

The water maze was a circular white pool $(1.5 \mathrm{~m}$ in diameter, $45 \mathrm{~cm}$ deep) filled to within $24 \mathrm{~cm}$ of the top with water at $29^{\circ} \pm 1^{\circ}$ and placed in a room with numerous distal cues. A floating layer of polypropylene pellets was used to make the water surface opaque and hide the location of the escape platform. Two acrylic escape platforms were available, each with a top $(15 \times 15 \mathrm{~cm})$ that was always $1.5 \mathrm{~cm}$ below the surface of the water. One contained a brick as a weight, and the other contained a large permanent magnet from a Grass Model 7 polygraph pen driver. Thick black curtains could be hung from a track mounted on the ceiling to completely surround the pool and occlude distal cues. Neither the ceiling nor the lighting provided spatial reference points, and the experimenter was not visible to the rat after the start of a trial. Training and probe trails were videotaped by a recessed camera above the pool, and swim paths were digitized, stored on disk, and analyzed by a computerized tracking system (Poly-Track, San Diego Instruments) as described previously (Cain, Saucier, Hall, Hargreaves, \& Boon, 1996).

\section{Procedure}

A training trial was begun by placing the rat into the water next to and facing the pool wall. The trial ended when the rat mounted the escape platform or when $60 \mathrm{sec}$ had elapsed, at which point the rat was placed on the platform by hand, where it remained for $15 \mathrm{sec}$. Placement into the pool for training trials was at NE, SE, SW, and NW in pseudorandom order. For probe trials, the escape platform was removed, and the rat was allowed to swim freely for $60 \mathrm{sec}$. The rat was placed under a heat lamp after each training or probe trial to maintain core temperature. The intertrial interval was $5 \mathrm{~min}$.

\section{Data Analysis}

Data were analyzed using one-way and repeated measures analysis of variance (ANOVA) and $t$ tests. Significance was reported when a $p$ value of .05 or less was obtained.

\section{EXPERIMENT 1}

Experiment 1 was designed to evaluate the role of proximal and distal cues, path integration, and magnetic field information by first evaluating whether rats trained to find an escape platform using distal cues could navigate to it if the distal cues were occluded during a probe trial. In the absence of distal cues, one possible basis for a place response might be cues contained within the pool itself(i.e., proximal cues). This possibility was tested by using a small black mark on the white wall of the pool that remained in a constant position relative to the escape platform throughout all training and probe trials (the proximal cue). The mark was intended to mimic the kind of cue that might result from paint chipping off the pool wall. Other possible sources of information might be path integration or magnetic field information (August et al., 1989; Burda et al., 1990; Chafetz, 1982; Dudchenko et al., 1997; Etienne, 1987; Etienne, Maurer, Saucy, \& Teroni, 1986; Etienne, Maurer, \& Seguinot, 1995; Kavaliers et al., 1993; Martin et al., 1997; Mather, 1985; Mather \& Baker, 1981; McNaughton et al., 1996; Olcese et al., 1988; Potegal, 1987; Whishaw et al., 1997).

After the completion of spatial training, several probe trials were conducted. For some of these, the black curtains were in place; for others, the black curtains were removed, allowing the rats to make use of distal cues in the room. Probe trials without black curtains were included to confirm that the rats had acquired the task during training and could exhibit the expected bias in swim time in the platform quadrant (Morris, 1984).

\section{Method}

\section{Training}

Two groups of rats (Group 1, $n=10$; Group 2, $n=10$ ) were trained for 2 days with 10 trials per day (no curtains) using the brickweighted escape platform at magnetic north, $15 \mathrm{~cm}$ from the wall. Measurements of the static magnetic field in each direction with a flux-gate-magnetometer (model FGM-3DI; Walker Scientific Inc., Worcester, MA) indicated a value of $45,000 \mathrm{nT}$ at the level of the platform, which closely approximates the expected magnetic field at the latitude of London, Ontario. Ambient $60-\mathrm{Hz}$ fields measured with a field monitor (ELF-66D; Walker Scientific) were approximately $0.3 \mu \mathrm{T}$ rms.

Throughout all training and probe trials, a proximal cue was available on the inside of the maze wall in constant relation to the escape platform at $30^{\circ}$ angular deviation from the platform position. The cue was a small $(10 \times 8 \times 6 \mathrm{~mm})$ triangular black mark, which contrasted strongly with the white pool wall. The black mark was clearly visible to human observers at a distance of more than $10 \mathrm{~m}$.

\section{Probe Trials}

Group 1: Role of proximal and distal cues, path integration, and magnetic field information. At the end of training on the 2 nd day, Group I was given four probe trials to evaluate place responses to the platform position. The rats were introduced into the pool at magnetic south for all probe trials. Probe Trials 1 and 3 were con- 
ducted with the black curtains in place around the pool. Probe Trials 2 and 4 were conducted with the curtains removed to confirm that place responses could be exhibited when distal cues were available (Morris, 1984).

Data from the first probe trial indicated that the rats in Group 1 exhibited a weak spatial bias for the platform quadrant (see Results section). This weak spatial bias could have resulted from the rats' knowledge of the point of entry into the pool (Dudchenko et al., 1997). Thus, if the rats knew the point of entry into the pool as they were being placed into it through an opening in the curtains, they would also know something about the location of the escape platform. Point of entry information could be based on information from distal cues, path integration, or both. During Probe Trial 1, Group 1 rats were carried to the pool by hand; thus, they could view the room cues while being carried to the pool. They could also make use of path integration mechanisms at the same time, if such mechanisms exist in the rat. To determine whether Group 1 rats made use of point of entry information, additional probe trials were conducted. First, Probe Trial 2 (no curtains) was conducted to confirm that a place response could be exhibited when distal cues were available, followed by two additional training trials (no curtains) to avoid possible extinction effects. This was followed by Probe Trials 3 and 4 . For Probe Trial 3, Group 1 was prevented from seeing the room cues, and path integration information was disrupted by carrying the rats to the pool in a covered box using a disorientation carrying procedure (Dudchenko et al., 1997; Knierim, Kudrimoti, \& McNaughton, 1995; Martin et al., 1997). The experimenter walked slowly around the pool twice, making one complete $\left(360^{\circ}\right)$ rotation during each circuit. He then leaned into the curtained area immediately above the pool and, with the curtains closed around him, opened the box and placed the rat into the water. Thus, Probe Trial 3 was intended to provide a test of the rats' ability to navigate in the absence of distal cue and path integration information. The rats then received Probe Trial 4 (no curtains; conventional carrying to the pool) to further confirm their ability to display a place response. After the completion of these procedures, the rats received another 4 days of standard maze training with no curtains and the escape platform at north. These data from the 6 days of training were used as control data for Experiment 2.

Group 2: Role of path integration. Group 2 was tested to evaluate whether the rats could display a place response without distal cues, using path integration information. Group 2 was spatially trained for 2 days and given two probe trials. For Probe Trial 1, black curtains were in place around the pool, and all lights in the testing room were extinguished from the time the rat was picked up in the holding box until it was released into the water. The rat was carried to the pool by hand during the lights-off period in the same manner as during spatial training (disorientation carrying procedure not used). The lights were then turned on. Thus, for Group 2, Probe Trial 1 was intended to evaluate the role of path integration. We expected that if Group 2 rats could make use of path integration, they would display a place response during Probe Trial 1 despite being transported from the holding box to the pool in complete darkness. Probe Trial 2 was conducted in the conventional manner with the room lights on and no black curtains.

\section{Results and Discussion}

\section{Escape Platform Search Time}

By the end of training on Day 2, mean escape time had declined to an asymptote of approximately $7 \mathrm{sec}$ for both groups, which did not differ ( $p>.05$; see Figure 1).

\section{Probe Trials}

Group 1: Role of proximal and distal cues, path integration, and magnetic field information. Mean platform quadrant search time of Group 1 differed across probe trials $[F(3,24)=7.0, p<.002$; see Figure 2 and Table 1$]$. Mean platform quadrant search time during Probe Trial 1 exceeded chance (independent two-tailed $t$ test, $t=2.1, p<$ $.05)$, but platform quadrant search time during Probe Trial 3 did not exceed chance $(p>.05)$. Thus, despite the presence of curtains around the pool, when the rats knew their point of entry into the pool during Probe Trial 1 (based on viewing the distal cues while being carried to the pool, or path integration, or both), their platform quadrant search time was significantly above chance. During Probe Trial 3, when information from these sources of information was not available, their platform quadrant search time was at chance, suggesting that magnetic field and proximal cue information was not used by the rats.

Probe Trials 2 and 4, which were conducted with distal cues in full view, confirmed robust place responses (Probe Trial 2, $t=8.4, p<.0001$; Probe Trial 4, $t=5.0, p<.0001$; see Figure 2 and Table 1). Thus, when all information was available, the rats performed robust place responses.

Group 2: Role of path integration. For Group 2, there was a nonsignificant trend for a difference between search times in Probe Trials 1 and $2(p<.07)$, and both values exceeded chance (Probe Trial 1, $t=3.1, p<.006$; Probe Trial 2, $t=4.6, p<.0001$; see Figure 2 and Table 1). Thus, the availability of path integration information in Probe Trial 1 supported above-chance platform quadrant search time in Group 2.

Initial trajectory of probe trial swims. Further analyses were carried out to evaluate performance during the early portion of the probe trials to evaluate the initial trajectory of the probe trial swims. The incidence of direct swims from the point of release to the point of contact with the escape platform (Whishaw \& Tomie, 1987), the latency to enter the platform quadrant, and platform quadrant search time during the first $15 \mathrm{sec}$ of the probe trials generally exhibited patterns similar to that of Group 1 in Figure 2 (data not shown). However, none of these measures differed across probe trials $(p>.05)$. Thus, the place responses displayed in Probe Trial 1 were weak and did not appear to be manifested during the early portion of the probe trial.

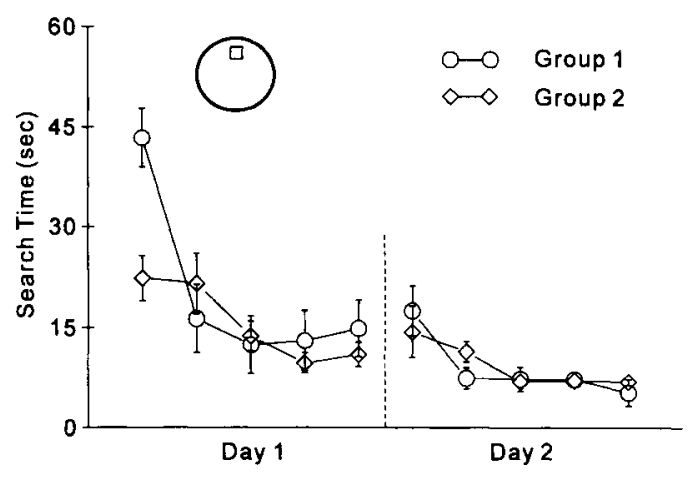

Figure 1. Water maze acquisition, Experiment 1. Escape platform search time graphed as blocks of two training trials. The insert illustrates the position of the escape platform during training. In all figures, data points represent mean $\pm S E M$. 


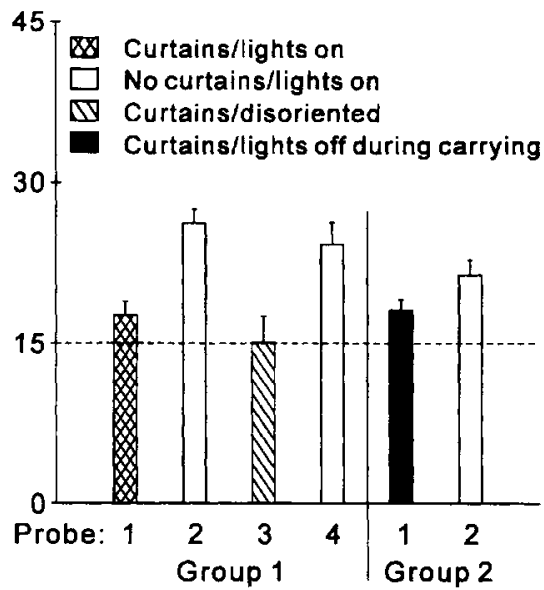

Figure 2. Platform quadrant search time, Experiment 1. The dotted line represents chance (15 sec). For Group 1, platform quadrant search time exceeded chance on Probe Trials 1, 2, and 4 when point of entry and path integration information (Probe 1) or distal visual cues and path integration information (Probes 2 and 4) were available (see Table 1). Platform quadrant search time failed to exceed chance when only magnetic field and proximal cue information was available (Probe 3 ). For Group 2, platform quadrant search time exceeded chance on Probe Trial 1 when path integration information was available and on Probe Trial 2 when distal visual cues and path integration information were available.

The results showed that Group 1 rats, which displayed robust place responses when all sources of information were available (Probe Trials 2 and 4), did not exhibit place responses during Probe Trial 3 , when information from distal cues and path integration information was occluded or disrupted. The absence of place responses during Probe Trial 3 implies that the rats were unable to navigate using the proximal cue or magnetic field information. The occurrence of place responses during Probe Trial 1 suggests that the rats made use of distal cue information while they were carried to the pool, or path integration, or both, to guide place responding. However, place responding during Probe Trial 1 was weak, relative to the place responding when distal visual cues were available during Probe Trials 2 and 4 . The occurrence of place responses by Group 2 rats during Probe Trial 1, in which the rats were carried to the pool in darkness, suggests that the basis for the place responses by Group 1 during Probe Trial 1 was very likely path integration information. In sum, the results were consistent with the use of distal visual cues for robust place responding and the use of path integration information for weak place responding, but there was no evidence that a proximal cue or magnetic field information was used to guide place responding.

\section{EXPERIMENT 2}

Although no evidence for navigation by a proximal cue or magnetic field information was obtained in Experiment 1 , the training conditions might not have been opti- mal because the rats were not required to learn to make use of these information sources during spatial training. Had the rats been trained without distal visual cues, they might have made use of the proximal cue or magnetic field information during both training and probe trials. Experiment 2 evaluated this possibility by giving rats 6 days of escape platform training with black curtains around the pool. They were prevented from obtaining information from visual cues while being carried to the pool or using path integration information during the first 6 days of testing by the disorientation carrying procedure from Experiment 1 .

\section{Method}

\section{Training, Days 1-6}

Naive rats $(n=6)$ were used. Training trials were similar to those of Experiment 1 except that black curtains were in place during training, and 6 days of training were given, for a total of 60 training trials. The rats were carried to the pool using the disorientation carrying procedure of Experiment 1 . Although there is no evidence that rats can make use of position information from stationary black curtains, the curtains were moved pseudorandomly after every two training trials to prevent the rats from gaining consistent position information from this source. The proximal cue described in Experiment 1 was present, and it served as a consistent cue to the platform position. The brick-weighted platform was at magnetic north during all training trials. The rats from Experiment 1, which were trained for a total of 6 days without curtains, served as controls.

\section{Probe Trials, Days 1-6}

Probe trials were carried out with the black curtains in place around the pool after every 20 training trials, on Days 2, 4, and 6. The

Table 1

Probe Trial Platform Quadrant Search Time

\begin{tabular}{|c|c|c|c|}
\hline Probe Trial & $\begin{array}{c}\text { Information } \\
\text { Sources Available }\end{array}$ & $\begin{array}{l}\text { Platform Quadrant } \\
\text { Search Time (sec) }\end{array}$ & $p$ \\
\hline \multicolumn{4}{|c|}{ Experiment 1 , Group $1(n=10)$} \\
\hline 1 & $\begin{array}{l}\text { Point of entry into pool, } \\
\text { Path integration }\end{array}$ & $17.7 \pm 1.8$ & $<.05$ \\
\hline 2 & All sources ( see text) & $26.2=1.3$ & $<.0001$ \\
\hline 3 & $\begin{array}{l}\text { Proximal cue, } \\
\text { Static magnetic field }\end{array}$ & $15.1 \pm 2.4$ & n.s. \\
\hline 4 & All sources & $24.3 \pm 1.8$ & $<.0001$ \\
\hline \multicolumn{4}{|c|}{ Experiment 1, Group $2(n=10)$} \\
\hline 1 & Path integration & $18.1 \pm 1.0$ & $<.006$ \\
\hline 2 & All sources & $21.4 \pm 1.4$ & $<.0001$ \\
\hline \multicolumn{4}{|c|}{ Experiment $2(n=6)$} \\
\hline 1 & $\begin{array}{l}\text { Proximal cue, } \\
\text { Static magnetic field }\end{array}$ & $14.1 \pm 1.4$ & n.s. \\
\hline 2 & $\begin{array}{l}\text { Proximal cue, } \\
\text { Static magnetic field }\end{array}$ & $16.1+0.5$ & \\
\hline 3 & $\begin{array}{l}\text { Proximal cue, } \\
\text { Static magnetic field }\end{array}$ & $156+0.9$ & n.s. \\
\hline 4 & All sources & $26.3 \pm 2.2$ & $<.001$ \\
\hline \multicolumn{4}{|c|}{ Experiment $3(n=6)$} \\
\hline Day 2 & Static magnetic field & $15.1 \pm 1.4$ & n.s. \\
\hline Day 4 & Static magnetic field & $19.3 \pm 2.2$ & n.s. \\
\hline Day 6 & Static magnetic field & $16.8 \pm 1.5$ & n.s. \\
\hline Day 7 & All sources & $22.7 \pm 3.2$ & $<.04$ \\
\hline Day 8 & All sources & $23.4 \pm 2.1$ & $<.002$ \\
\hline
\end{tabular}

Note - n.s. $=$ not significant; $\mathrm{sec}=$ seconds. Values are mean $\pm S E M$. For $p$, chance $=15$ sec. 


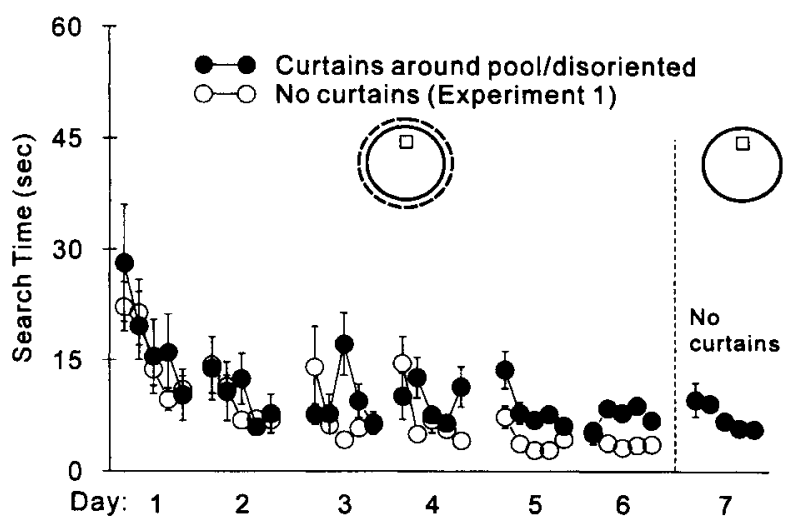

Figure 3. Water maze acquisition, Experiment 2. Escape platform search time graphed as blocks of two training trials. Search time for the rats trained with curtains around the pool and brought to the pool using a disorientation carrying method declined to $8.03 \pm 0.83$ sec on the last 7 trials on Day 6. On Day 7 , when distal cues were available, search time declined to $6.23 \pm$ $0.64 \mathrm{sec}$ on the last 7 trials. The inserts illustrate the pool, the presence or absence of curtains (indicated by a dotted circle), and the position of the escape platform during training.

rats were carried to the pool for probe trials using the disorientation carrying procedure from Experiment 1 . There was no platform in the pool.

\section{Training and Probe Trials, Day 7}

On Day 7, the curtains were removed, and the rats were given 10 training trials during which they could make use of distal cues in the room, in order to confirm that they could navigate accurately when distal cues were available. The escape platform was at magnetic north. At the end of training on Day 7 , the platform was removed, and a probe trial was given (no curtains). The rats were carried to the pool conventionally (disorientation carrying procedure not used).

\section{Results and Discussion}

\section{Escape Platform Search Time}

By the end of training on Day 6, escape platform search time for the control rats was approximately $4 \mathrm{sec}$, and that for the rats trained with distal cues occluded was approximately $8 \mathrm{sec}$ (Figure 3). Escape platform search time during Days 1-6 was longer for the rats trained with distal cues occluded than for controls $[F(1,70)=8.4, p<.01]$. During this time, the rats trained with distal cues occluded by curtains swam around the pool at a distance from the wall that made rapid contact with the escape platform likely (Whishaw \& Jarrard, 1995; Whishaw \& Tomie, 1987).

On Day 7 , when the rats that were previously trained with curtains could make use of distal cues, mean search time appeared to decline further. To examine this, search times from the last 7 training trials on Days 6 and 7 were compared. The last 7 trials were used to allow the rats to learn the position of the escape platform on Day 7 during the first 3 training trials with visual cues available before making statistical comparisons. Mean search time declined from Day 6 to Day $7[F(1,4)=9.6, p<.04]$.

\section{Probe Trials}

Can rats use proximal cue or magnetic field information when distal cues and path integration are unavailable? Probe trials indicated chance-level platform quadrant search times on Days 2, 4, and 6 (Probe Trials 1-3) when distal cues and path integration information were unavailable, but above-chance search times on Day 7 (Probe Trial 4) when distal cues and path integration information were available $[F(3,12)=12.1, p<.001$; Probe Trials $1-3$ vs. chance, each $p>.05$; Probe Trial 4 vs. chance, $t=5.0, p<.001$; see Figure 4 and Table 1]. During Day 7, the rats swam more directly to the escape platform after the first few trials. The incidence of direct swims increased from $2.4 \%$ during the last 7 training trials on Day 6 to $21.4 \%$ during the last 7 training trials on Day $7[F(1,4)=12.8, p<.03]$.

The results indicate the development of a looping swim strategy during training with curtains on Days 1-6 that accounted for the shortening of search times (Whishaw \& Jarrard, 1995; Whishaw \& Tomie, 1987). There was no evidence of place responding during Days 1-6 when distal cues and path integration were unavailable. In contrast, on Day 7 when distal cues and path integration were available, escape platform search time declined further, and place responding was evident. Thus, despite the occlusion of distal cues and disruption of path integration during extensive training, the results yielded no evidence of place responding during Days $1-6$. More extensive training might have provided such evidence, but the extent of such training would have exceeded the amount of training required for the adoption of a place navigation strategy using distal cues in typical water maze tasks or for the adoption of a path integration strategy as found in Experiment 1 . These results imply that the rats did not make use of proximal cue or magnetic field information for place

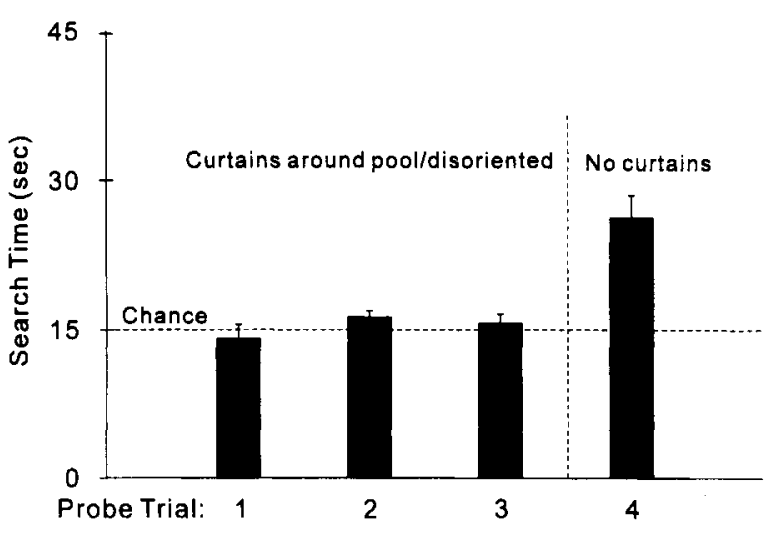

Figure 4. Platform quadrant search time, Experiment 2. Probe Trials 1-3 occurred after every 20 training trials on Days 2,4 , and 6. The rats were carried to the pool for training on Days $1-6$ and for Probe Trials $1-3$ in a small box using the disorientation procedure described in Experiment 1 . Probe Trial 4 occurred on Day 7 with distal visual cues available and no disorientation, after 10 training trials without curtains. 


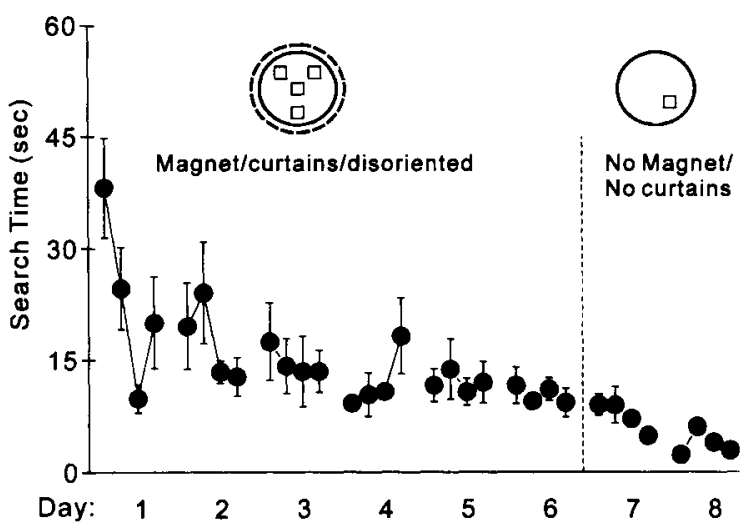

Figure 5. Water maze acquisition, Experiment 3. Escape platform search time graphed as blocks of two training trials. Training on Days 1-6 was carried out with distal visual cues occluded by curtains, a disorientation carrying procedure, and the magnetweighted platform placed pseudorandomly in the positions indicated on the insert. Training on Days 7 and 8 was carried out with distal cues visible; it made use of the brick-weighted platform placed in the position indicated on the insert. The inserts illustrate the pool, the presence or absence of curtains (indicated by a dotted circle), and the position of the escape platform. During training on Days 1-6 the platform was moved pseudorandomly to one of the four positions shown after every trial.

responding during extensive training when no other information was available for spatial navigation.

\section{EXPERIMENT 3}

Experiments 1 and 2 yielded no evidence for the use of magnetic field information, but it is possible that the magnetic field in the water maze room was not optimal for use in navigation. Therefore, Experiment 3 further evaluated the possibility of navigation by magnetic fields by making use of direction-static magnetic field information generated by a permanent magnet contained within the escape platform itself.

\section{Method}

\section{Training, Days 1-6}

Naive rats $(n=6)$ were used. The procedure was similar to that of Experiment 2 except that the escape platform containing the permanent magnet was used, and the platform was moved in a predetermined position from trial to trial to make successful performance contingent on use of the magnetic field generated by the platform magnet. Thus, the rats could determine the location of the escape platform on any particular trial only by using the static magnetic field gradient and presumed directional information provided by the magnet. The platform arrangement of Whishaw (1985) was used because it provided for four varied positions in the pool: near the wall, in the middle of the pool, and two locations intermediate between these extremes. Eight training trials per day were given in pseudorandom order (two per position). During training trials on Days 1-6, black curtains surrounded the pool, and the rats were carried to the pool using the disorientation carrying method from Experiment 1, in order to prevent the use of dis- tal cues during carrying or path integration information. Measurements of static magnetic field strength indicated a value of approximately $200,000 \mathrm{nT}$ at the top surface of the platform.

\section{Probe Trials, Days 1-6}

Probe trials were carried out after every 16 training trials (Days 2, 4 , and 6), with curtains around the pool. For probe trials, the escape platform was removed, and the magnet alone was positioned at the bottom of the pool in the center of the following quadrants: Day 2 , SE; Day 4, NW; Day 6, NE. Thus, the rats had to make use of the static magnetic field to perform a place response.

\section{Training and Probe Trials, Days 7-8}

On Days 7 and 8, the black curtains were removed, and the rats were given 8 training trials per day during which they could make use of distal cues in the room, in order to confirm that they could navigate accurately in the maze when distal cues were available. For these trials, the brick-weighted escape platform was placed at SE. At the end of Days 7 and 8 , the rats were given a probe trial with the platform removed.

\section{Results and Discussion}

\section{Escape Platform Search Time, Days 1-6}

Escape platform search time declined to approximately $10 \mathrm{sec}$ by Day 6 (see Figure 5). The rats adopted a looping search strategy similar to that of Experiment 2, which quickly brought them into contact with the platform but produced few direct swims. On Days 7 and 8, when the rats could make use of distal cues, platform search times decreased further [Day 6 vs. Day $7, F(1,4)=15.3, p<.02$; Day 6 vs. Day $8, F(1,4)=258.3, p<.001]$.

\section{Probe Trials}

Can rats use direction-static magnetic field information to navigate in the water maze? On Days 2,4 , and 6 , platform quadrant search times were not significantly above chance [Days $2-8$ overall, $F(4,16)=4.5, p<.02$; Days 2 , 4 , and 6 vs. chance, $p>.05$; see Figure 6 and Table 1], and there were few direct swims (range of daily mean percent direct swims, $10.4 \%-14.6 \%$ ). On Days 7 and 8 , when the rats could make use of distal cues and path integration information, platform quadrant search times increased to above-chance levels (Day 7 vs. chance, $t=2.4, p<.04$; Day 8 vs. chance, $t=4.0, p<.002)$. Similarly, the percents of direct swims on Day $7(32.0 \%)$ and Day $8(66.0 \%)$ were greater than on Day $6(12.5 \%)$ [Day 6 vs. Day $7, F(1,4)=$ $38.6, p<.005$; Day 6 vs. Day $8, F(1,4)=111.5, p<.001]$.

The results yielded no evidence that the rats trained with a magnet in the escape platform learned to swim to it effectively when other cues were not available. Platform quadrant search time was not different from chance, and percents direct swims were low and comparable to values obtained from a group of random platform control rats trained in the same apparatus (Cain et al., 1996). When distal cues were made available by the removal of the black curtains, the rats made rapid improvements in all three measures. More extensive training or a stronger magnet might have provided evidence of place responding based 


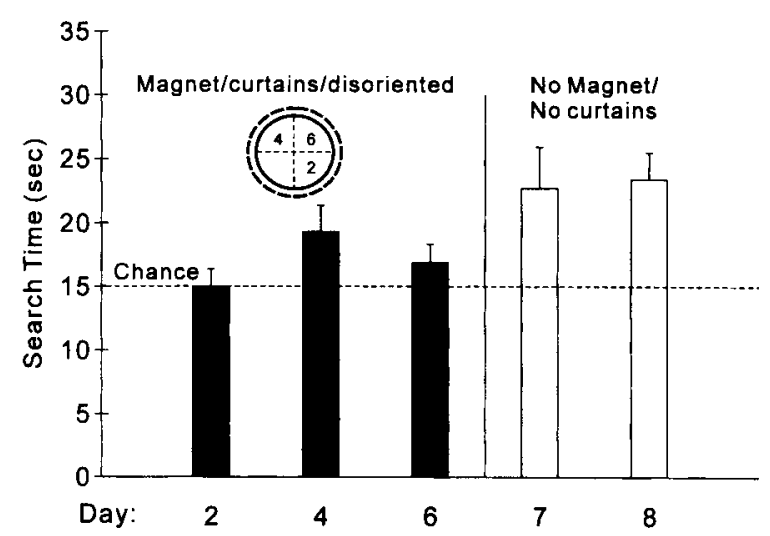

Figure 6. Platform quadrant search time, Experiment 3. The first three probe trials occurred after training on Days 2,4 , and 6. Distal visual cues were occluded by curtains (indicated by a dotted circle on the insert), and the magnet was placed on the bottom of the pool in the positions indicated on the insert for Days 2 , 4, and 6. The last two probe trials occurred after training on Days 7 and 8. Distal visual cues were visible, and there was no magnet or platform in the pool.

on the local magnetic field, but more extended training would exceed the amount of training required for place navigation using distal cues in typical water maze tasks.

\section{GENERAL DISCUSSION}

The results provided no evidence for place responding by proximal cue (Experiments 1 and 2 ) or static magnetic field information (Experiments 1-3), but they did provide evidence for place responding based on distal visual cues and path integration (Experiments 1-3). Experiment 1 also provided evidence for place responding based on path integration by itself, when distal visual cues were not available.

There was no evidence that the proximal cue supported place responding, even when distal visual cues were occluded and extensive training was given. This suggested that the proximal cue was not salient enough to serve as a guide to navigation, despite the fact that it contrasted sharply with the white maze wall and was clearly visible to human observers at $>10 \mathrm{~m}$. The fact that a large $(14 \times$ $18.5 \mathrm{~cm}$ ) landmark cue attached to the pool wall immediately adjacent to a moving escape platform, which remained in constant relation to the platform when it was moved, supported effective navigation (Kolb et al., 1994)-and the fact that our much smaller proximal cue at a $30^{\circ}$ deviation from the platform position did not-suggests that landmark size and position may be important variables. However, the Kolb et al. study differed from ours in that distal cues were visible but irrelevant as cues for navigation because the platform and large landmark cue were moved between trials. In Experiment 1 of the present study, the platform remained in one position and distal cues were visible during training, making the distal cues highly relevant for navigation purposes, and the proximal cue presumably less so. Thus, movement of the large landmark cue with the platform and the irrelevance of distal visual cues for navigation purposes in the Kolb et al. study may have been important. We attempted to make our proximal cue maximally relevant for navigation by giving extensive training with distal visual cues occluded by curtains in Experiment 2; the rats nevertheless failed to use the proximal cue as a guide to navigation. This suggests that small blemish marks on the pool wall may not be used for navigation in water maze studies.

There is good evidence for the use of magnetic field information in navigation by some nonmammalian species (Wiltschko \& Wiltschko, 1996), but the evidence for its use by mammals is inconsistent; some researchers have obtained no evidence for its use by rodents (Bovet, Dolivo, George, \& Gogniat, 1988; Etienne et al., 1986; Lovegrove, Kortner, \& Kortner, 1992; Madden \& Phillips, 1987). We found no evidence that the static magnetic field provided either directional or position information in Experiment 2 when the use of other sources of information was prevented during training or in Experiment 3 when a magnet was placed at the bottom of the pool during training and probe trials. However, in comparison with the conditions of large-scale migratory navigation (Wiltschko \& Wiltschko, 1996), the water maze task involves very short distances, and it is possible that the magnetic field information is too imprecise to support accurate navigation in this task. Whether the earth's magnetic field may provide directional or positional information to rats navigating over longer distances or in the wild is unknown, and our findings do not preclude this possibility.

Previous work has shown that mammals can use path integration to navigate in radial arm maze and food-carrying tasks (Dudchenko et al., 1997; Etienne, 1987; Martin et al., 1997; Whishaw et al., 1997). The results of Experiment 1 extend this conclusion to the water maze and provide the first evidence that path integration information by itself can support place responding in the water maze. However, path integration information by itself was less accurate than when both distal visual cue and path integration information was available.

In sum, we found no evidence for place responding based on proximal cue or static magnetic field information in a water maze task. Distal visual cues led to highly reliable place responding, and path integration led to weak but reliable place responding. The robust place responding when both distal visual cues and path integration were available is consistent with the suggestion that rats use numerous navigation strategies concurrently in the water maze (Whishaw \& Mittleman, 1986).

\section{REFERENCES}

August, P. V., Ayvazian, S. G., \& Anderson, J. G. T. (1989). Magnetic orientation in a small mammal Peromyscus leucopus. Journal of Mammalogy, 70, 1-9.

Bovet, J., Dolivo, M., George, C., \& Gogniat, A. (1988). Homing behavior of wood mice (Apodemus) in a geomagnetic anomaly. Saugetierkunde, 53, 333-340. 
Burda, H., Marhold, S., Westenberger, T., Wiltschko, R., \& Wiltschko, W. (1990). Magnetic compass orientation in the subterranean rodent Cryptomys hottentotus (Bathyergidae). Experientia, 46, $528-530$

Cain, D. P., Saucier, D., Hall, J., Hargreaves, E. L., \& Boon, F. (1996). Detailed behavioral analysis of water maze acquisition under APV or CNQX: Contribution of sensorimotor disturbances to druginduced acquisition deficits. Behavioral Neuroscience, 110, 86-102.

ChafeTz, M. D. (1982). Geomagnetic orienting in the radial eight-arm maze? Journal of General Psychology, 107, 287-295.

Dudchenko, P. A., Goodridge, J. P., Seiterle, D. A., \& Taube, J. S. (1997). Effects of repeated disorientation on the acquisition of spatial tasks in rats: Dissociation between the appetitive radial arm maze and aversive water maze. Journal of Experimental Psychology, 23, 194-210.

ETIENNE, A. S. (1987). The control of short-distance homing in the golden hamster. In P. Ellen \& C. Thinus-Blanc (Eds.), Cognitive processes in spatial orientation in animal and man (pp. 223-251). Dordrecht: Martinus Nijhoff.

Etienne, A. S., Maurer, R., Saucy, F., \& Teroni, E. (1986). Shortdistance homing in the golden hamster after a passive outward journey. Animal Behaviour, 34, 696-715.

Etienne, A. S., Maurer, R., \& Seguinor, V. (1995). Path integration in mammals and its interaction with visual landmarks. Journal of Experimental Biology, 199, 201-209.

Gallistel, C. R. (1990). The organization of learning. Cambridge, MA: MIT Press.

Kavaliers, M., Eckel, L. A., \& Ossenkopp, K.-P. (1993). Brief exposure to $60 \mathrm{~Hz}$ magnetic fields improves sexually dimorphic spatial learning performance in the meadow vole, Microtus pennsylvanicus. Journal of Comparative Physiology $A, 173,241-248$.

Kavaliers, M., \& Galea, L. (1994). Spatial water maze learning using celestial cues by the meadow vole, Microfus pennsylvanicus. Behavioural Brain Research, 61, 97-100.

Kavaliers, M., Ossenkopp, K.-P., Prato, F. S., Innes, D. G. L., Galea L. A. M., Kinsel la, D. M., \& Perrot, T. S. (1996). Spatial learning in deer mice: Sex differences and the effects of endogenous opioids and $60 \mathrm{~Hz}$ magnetic fields. Journal of Comparative Physiology A, $179,715-724$

Knierim, J. J., Kudrimoti, H. S., \& McNaughton, B. L. (1995). Place cells, head direction cells, and the learning of landmark stability. Journal of Neuroscience, 15, 1648-1659.

Kolb, B., Buhrmann, K., McDonald, R., \& Sutherland, R. J. (1994). Dissociation of the medial prefrontal, posterior parietal, and posterior temporal cortex for spatial navigation and recognition memory in the rat. Cerebral Cortex, 6, 664-680.

Lovegrove, B. G., Kortner, G., \& Kortner, G. (1992). The magnetic compass orientation of the burrows of the Damara mole-rat Cryptomys damariensis (Bathyergidae). Journal of Zoology, 226, 631-633.

Madden, R. C., \& Phillips, J. B. (1987). An attempt to demonstrate magnetic compass orientation in two species of mammals. Animal Learning \& Behavior, 15, 130-134.

Martin, G. M., Harley, C. W., Smith, A. R., Hoyles, E. S., \& Hynes, C. A. (1997). Spatial disorientation blocks goal location on a plus maze but does not prevent goal location in the Morris maze. Journal of Experimental Psychology, 23, 183-193.

MATHER, J. G. (1985). Magnetoreception and the search for magnetic material in rodents. In J. L. Kirschvink, D. S. Jones, \& B. J. Macfadden (Eds.), Magnetic biomineralization and magnetoreception in organisms (pp. 509-533). New York: Plenum.

Mather, J. G., \& BAKer, R. R. (1981). Magnetic sense of direction in woodmice for route-based navigation. Nature, 291, 152-155.

McNaughton, B. L., Barnes, C. A., Gerrard, J. L., Gothard, K., Jung, M. W., Knierim, J. J., Kudrimoti, H., Qin, Y., Skaggs, W. E., Suster, M., \& Weaver, K. L. (1996). Deciphering the hippocampal polyglot: The hippocampus as a path integration system. Journal of Experimental Biology, 199, 173-185.

McNaughton, B. L., Chen, L. L., \& Markus, E. J. (1991). "Dead reckoning," landmark learning, and the sense of direction: A neurophysiological and computational hypothesis. Journal of Cognitive Neuroscience, 3, 190-202.

Morris, R. G. M. (1981). Spatial localization does not require the presence of local cues. Learning \& Motivation, 12, 239-260.

MorRis, R. G. M. (1984). Developments of a water maze procedure for studying spatial learning in the rat. Journal of Neuroscience Methods, 11, 47-60.

OlCese, J., Reuss, S., \& SEMM, P. (1988). Geomagnetic field detection in rodents. Life Sciences, 42, 605-613.

Potegal, M. (1987). The vestibular navigation hypothesis: A progress report. In P. Ellen \& C. Thinus-Blanc (Eds.), Cognitive processes in spatial orientation in animal and man (pp. 28-34). Dordrecht: Martinus Nijhoff.

Sutherland, R. J., Whishaw, I. Q., \& Regehr, J. C. (1982). Cholinergic receptor blockade impairs spatial localization by use of distal cues in the rat. Journal of Comparative \& Physiological Psychology, 96, 563-573.

WhishaW, I. Q. (1985). Formation of a place learning-set by the rat: A new paradigm for neurobehavioral studies. Physiology \& Behavior, 35, 139-143.

WhishaW, I. Q., \& JARRARD, L. E. (1995). Similarities vs. differences in place learning and circadian activity in rats after fimbria-fornix section or ibotenate removal of hippocampal cells. Hippocampus, 5, 595-604.

Whishaw, I. Q., McKenna, J. E., \& MaAswinkel, H. (1997). Hippocampal lesions and path integration. Current Opinion in Neurobiology, 7, 228-234.

Whishaw, I. Q., \& Mittleman, G. (1986). Visits to starts, routes, and places by rats (Rattus norvegicus) in swimming pool navigation tasks. Journal of Comparative Psychology, 100, 422-431.

WhishaW, I. Q., \& Tomie, J. (1987). Cholinergic receptor blockade produces impairments in a sensorimotor subsystem for place navigation in the rat: Evidence from sensory, motor, and acquisition tests in a swimming pool. Behavioral Neuroscience, 101, 603-616.

WiltschKo, W., \& WilTSCHKo, R. (Eds.) (1996). Magnetic orientation in animals (Zoophysiology, Vol. 33). New York: Springer-Verlag.

(Manuscript received June 18, 1997; revision accepted for publication September 17, 1997.) 